\title{
Assessing Traumatic Experiences of Justice-Involved Latinx Youth: A Systematic Review
}

\author{
David Hoskins, Psy.D., M.A.S. ${ }^{1,2^{*}}$ \\ ${ }^{1}$ University of California San Francisco (UCSF), Center for the Vulnerable Child, USA \\ ${ }^{2}$ Alliant International University, USA
}

*Corresponding author: David Hoskins, Psy.D., M.A.S., University of California San Francisco (UCSF), Center for the Vulnerable Child; Alliant International University, San Francisco, California, USA

\begin{abstract}
Background: Traumatic experiences have been linked to the unmet behavioral health needs of justice-involved youth. Unmet behavioral health needs are some of the most salient predictors of initial justice involvement and recidivism. Latinx youth are one of the most overrepresented ethnic and racial minority groups in the Juvenile Justice System in the United States.
\end{abstract}

Main text: The primary aim of this systematic review was focused on justice-involved, Latinx youth in order to compare traumatic experiences and PTSD rates by race/ethnicity and gender.

Methods: For inclusion in this systematic review, studies had to be (a) Observational or randomized controlled trials,

(b) Assess either trauma exposure or symptoms of justice-involved Latinx youth 13 to 18 years of age, (c) Sample detained youth in the juvenile justice system, and (d) Compare Latinx trauma experience or symptoms by gender and/ or by ethnicity/race.

Results: There were differences in the number and types of traumatic experiences that the youth were asked about, which precluded allowing direct data comparisons across studies. Most of the studies varied in their measurement of PTSD. Trauma exposure rates by race/ethnicity and Latinx gender was mixed. Latinx youth disclosed PTSD symptoms at a statistically significant higher rate than did White and Black youth.

Conclusion: Trauma rate differences varied by study, presumably related to a lack of construct equivalence and variability in the number and types of traumatic experiences assessed by the measures. Further research is needed to inform appropriate assessment and treatment of justice-involved Latinx youth.

\section{Keywords}

Latinx, Juvenile Justice System, Trauma, Traumatic experiences, PTSD

\section{Introduction}

Latin ${ }^{1}$ youth who are involved in the juvenile justice system have been shown to have high rates of psychiatric need, substance use, and sexual risk behavior when compared to other racial and ethnic groups [1,2].

Overrepresentation among ethnic and racial groups at every intercept of the juvenile justice system is an alarming trend in this community [2]. For example, Latinx youth have been shown to be 2 to 3 times more likely to be incarcerated than non-Latinx White youth $[3,4]$ despite coming from an overall U.S. population that is less than one third the size of the White population. In addition, one third of Latinx Americans are under age 18 years, and the Latinx community is one of the largest and fastest growing ethnic groups in the United States [5], suggesting that these concerning trends may worsen.

\section{Trauma}

Research has shown that substance use is one of the most salient predictors of initial justice involvement and reoffending $[6,7]$. Psychiatric symptoms and sexual risk behaviors often co-occur with substance use [8] and exacerbate psychiatric symptoms of justice-involved youth [9]. The Substance Abuse and Mental Health Services Administration [10] linked psychiatric symptoms and substance use to a common environmental factor:

${ }^{1}$ Latinx is used throughout this paper to avoid gender binaries and to be inclusive of gender-fluid Latinx youth. The term encompasses a heterogenous population that differs in terms of race, social class, country of origin, and gender identity, to name a few (Vélez, 2016). 
Trauma exposure. In more recent years, researchers have differentiated trauma exposure and subsequent Posttraumatic Stress Disorder (PTSD) severity [11,12], noting that the diagnosis of PTSD stems from Vietnam War veterans and may not capture the full range of traumatic experiences and symptomatology that youth of color from low-income communities experience. Moreover, the number of traumas and distinct typologies of the traumatic experiences have recently become of interest to researchers who assess low-income communities or ethnic and racial minorities [13].

\section{Gender-Specific Comparisons across Race/ Ethnicity}

Much of the research on juvenile justice involvement that examines race and gender has investigated them as separate entities. However, intersectionality theorists postulate that it is important to analyze race and gender simultaneously. For example, behavioral health need patterns may differ between White females and Latinx females or between White males and Latinx males $[14,15]$.

In terms of justice-involved youth and their behavioral health needs, research has shown that Latinx males have rates of substance use disorders and affective disorders comparable to those of White males and higher than those of Black males [16] as well as earlier onset of drug use and more poly substance use [17] than for White and Black males. Justice-involved Latinx females have been shown to have more severe psychiatric symptoms than their White and Black counterparts [18] and heavier substance use than their Black and White counterparts [19].

The existence of gender differences among justice-involved youth is well understood [18]. Latinx males and females have been documented to differ in terms of their arrest rates, offense types, psychiatric symptoms, and substance use [18]. Arrest data collected from 5,400 law enforcement agencies throughout the United States showed that females make up 30\% of Latinx juvenile arrests (Office of Juvenile Justice and Delinquency Prevention). However, among Latinx youth who were arrested, females were arrested for $13.8 \%$ of violent crimes (i.e., murder, violent sex offense, robbery, and assault), $39.2 \%$ of larceny arrests, and $57.5 \%$ of runaway arrests (Office of Juvenile Justice and Delinquency Prevention). Research has also shown that justice-involved Latinx females have more severe internalizing disorders [20] and lower rates of substance detected by urinalysis than Latinx males (46.1\% versus 58.9\%; [1]). What still needs investigation is the underlying cause of these differences in the incarcerated Latinx youth population and how these underlying causes are related to their behavioral health needs (e.g., traumatic experiences).

Griner and Smith [21], in their seminal meta-analytic review, showed that culturally adapted mental health interventions were 4 times more effective when tailored to one cultural group versus to clients from a variety of cultural backgrounds. It has been documented that White youth prior to and upon contact with the justice system receive psychiatric services at much higher rates than do Latinx youth [22], even when the behavioral health needs are equivalent to those of other ethnicities and races [2]. Rather than basing all services on the needs of White youth, research that provides a nuanced understanding of trauma exposure as it relates to the Latinx community, and by gender in particular, is warranted.

\section{Study Aim}

In addition to Latinx youth being overrepresented at every intercept of the justice system [2], they have been shown to have unmet behavioral health needs [20]. Trauma has also been shown to be an environmental condition that disproportionately affects Latinx youth [23]. I address published research on trauma and PTSD in the juvenile justice system in this systematic review. Specifically, I asked this research question: Among justice-involved, detained youth in the United States, how do rates of trauma exposure and trauma symptoms differ by race, ethnicity, and gender? I hypothesized that detained Latinx youth in the United States will have higher rates of trauma exposure and trauma symptoms than both their White and Black peers. I also hypothesized that Latinx females will have higher rates of trauma exposure and trauma symptoms than Latinx males.

\section{Methods}

\section{Inclusion/Exclusion criteria}

To ensure methodological rigor and reduce bias, I developed a protocol to outline criteria for study inclusion and to identify relevant data for extraction. For inclusion in this systematic review, studies had to be (a) Observational or randomized controlled trials, (b) Assess either trauma exposure or symptoms of justice-involved Latinx youth 13 to 18 years of age, (c) Sample detained youth in the juvenile justice system, and (d) Compare Latinx trauma experience or symptoms by gender and/or by ethnicity/race. Assessment measure types included structured or semistructured measures of trauma exposure or symptoms. Exclusions included adult samples, case studies with a small sample size $(N<$ $10)$, non-detained and non-juvenile-justice population, Latinx not included in the analysis, non-U.S. population, and not able to compare either trauma exposure or severity of trauma symptoms.

\section{Search strategy}

Initially, I consulted seminal articles in the field of juvenilejustice to identify subject headings and keyword terms from titles and abstracts. A librarian at the University of California-San Francisco was consulted to identify additional subject headings and keywords and assemble 
search strings. Three databases-PsycINFO, PubMed, and EMBASE-were selected because of their scope, breadth, and expansive access for the topic's related disciplines. Search strings were run in these databases using the selected subject headings and keywords to identify the broadest set of relevant terms used for studies of Latinx youth and the juvenile justice population.

The following search strings were used in the three selected databases: PubMed: (justice-involved OR detained OR incarcerated OR first contact OR "justice-involved youth") AND (trauma OR psychotrauma OR "traumatic stress" OR "traumatic disorder" OR "traumatic exposure" OR "posttraumatic stress" OR "trauma symptoms" OR "stress disorders, post-traumatic" [MeSH]) AND (Latino OR Latina OR Hispanic OR Mexican OR immigrant) AND (youth OR "young adult" OR teen Or teens OR teenager OR teenagers OR adolescent OR adolescents OR adolescence). This search returned 58 results. In EMBASE, I used the following search strings: ('justice involved' OR detained OR incarcerated OR 'first contact' OR (first AND ('contact'/exp OR contact)) OR 'justice-involved youth') AND ('Hispanic'/exp OR Hispanic) AND ('PTSD'/exp OR PTSD OR 'traumatic experience'/exp OR 'traumatic experience'). This search returned 11 results. In PsycINFO, I used (justice-involved OR detained OR incarcerated OR first contact OR "justice-involved youth") AND (trauma OR psychotrauma OR "traumatic stress" OR "traumatic disorder "OR" traumatic exposure" OR "posttraumatic stress" OR "trauma symptoms" OR PTSD) AND (Latino OR Latina OR Hispanic OR Mexican OR immigrant). This search returned 20 results.

\section{Data abstraction, quality assessment, and analysis plan}

I independently abstracted the study data and as- sessed the quality of the identified studies. I undertook a narrative synthesis of the findings. Of the 89 references screened, 17 were duplicates, leaving a total of 72 unique papers. I then reviewed titles, abstracts, and full articles for study inclusion. I excluded the following: 23 studies that were not restricted to justice-involved youth, 15 studies that used adult samples, nine studies that did not involve comparisons to Latinx youth, seven studies on non-U.S. populations, six studies in which trauma rates were not discernable by race/ethnicity and/or PTSD rates were not discernable by race/ ethnicity or gender, and five case studies with small sample sizes (i.e., $N<5$ ). This left sevenstudies that fit the study criteria: [24-30]. I calculated odds ratios and standard deviations for each study. When the study authors provided the proportion and sample sizes, I used the Cornfield approximation in Stata 15.2 to calculate odds ratios and the $95 \%$ confidence intervals. When the mean and standard deviation were provided, I used the Practical Effect Size Calculator [31].

\section{Results}

\section{Participant characteristics}

Participants in all seven studies were enrolled after 1995. Combined, the study authors observed 94,103 detained youth in the juvenile justice system. Sample size in individual studies ranged from 264 to 64,329 . Latinx youth represented $9 \%$ to $30 \%$ of the samples. Of the seven studies that met inclusion criteria, youth represented three different intercepts of the juvenile justice system: (a) Pretrial detention $(n=5)$, incarcerated $(n=1)$, and repeat offenders $(n=1)$. All studies included a mix of ethnicities/races (i.e., Black, White, and Latinx) and genders; males were a majority in each study. Table 1 is a summary of all seven studies.

Table 1: Study summaries.

\begin{tabular}{|c|c|c|c|c|c|c|c|}
\hline Study & Location & $\begin{array}{l}\text { Justice } \\
\text { intercept }\end{array}$ & Total $N$ & Latinx $n, \%$ & $\begin{array}{l}\text { Trauma } \\
\text { exposure } \\
\text { measure }\end{array}$ & $\begin{array}{l}n \text { of traumas } \\
\text { elicited }\end{array}$ & $\begin{array}{l}\text { Posttraumatic } \\
\text { stress disorder } \\
\text { measure }\end{array}$ \\
\hline $\begin{array}{l}\text { Abram, et al. } \\
\text { [24] }\end{array}$ & Illinois & Pretrial detention & 898 & $252(28)$ & DISC-IV & 8 & DISC-IV \\
\hline $\begin{array}{l}\text { Baglivio, et al. } \\
\text { [25] }\end{array}$ & Florida & Repeat offenders & 64,329 & $9,906(15.4)$ & ACES & 10 & \\
\hline $\begin{array}{l}\text { Ford, et al. } \\
\text { [26] }\end{array}$ & Connecticut & Pretrial detention & 264 & $79(30)$ & TESI & 7 & UCLA PTSD-RI \\
\hline $\begin{array}{l}\text { Ford. et al. } \\
\text { [27] }\end{array}$ & Connecticut & Pretrial detention & 1,959 & $587(30)$ & TESI & 21 & UCLA PTSD-RI \\
\hline $\begin{array}{l}\text { King, et al. } \\
\text { [28] }\end{array}$ & Illinois & Pretrial detention & 1,735 & $488(28)$ & $\begin{array}{l}\text { Self-report/ } \\
\text { official records } \\
\text { of abuse }\end{array}$ & 11 & \\
\hline $\begin{array}{l}\text { Lau, et al. } \\
\text { [29] }\end{array}$ & $\begin{array}{l}\text { Midwestern } \\
\text { United States }\end{array}$ & Pretrial detention & 23,831 & $831(9.8)$ & MAYSI & 4 & \\
\hline $\begin{array}{l}\text { Rawal, et al. } \\
\text { [30] }\end{array}$ & Illinois & Incarcerated & 370 & $67(18.1)$ & $\begin{array}{l}\text { Official } \\
\text { records }\end{array}$ & 3 & \\
\hline
\end{tabular}

Note: DISC-IV = National Institute of Mental Health Diagnostic Interview Schedule for Children, Version IV; ACES = Adverse Childhood Experiences; Traumatic Events Screening Inventory; Massachusetts Youth Screening Instrument; UCLA PTSD RI = UCLA Post-Traumatic Stress Disorder Reaction Index. 


\section{Measurement of trauma experience}

There were differences in the number and types of traumatic experiences that the youth were asked about, which precluded allowing direct data comparisons across studies. At the lower end, four studies queried for eight or fewer traumatic experiences $[24,26,29,30]$ versus three that queried for 10 or more $[25,27,28] \min =3$ [30], $\max =21$ [27]. Even when two articles $[26,27]$ used the same measure (i.e., the Traumatic Events Screening Inventory) to query trauma exposure, the difference between the number of traumatic exposures queried was 14. A traumatic exposure that was not commonly assessed for wastraumatic separation or loss due to immigration $[24-26,29,30]$.

\section{Measurement of PTSD}

Most of the studies varied in their measurement of PTSD. Only two studies compared PTSD symptom severity across races/ethnicities $[24,26]$ and only one study compared PTSD severity by Latinx gender [24]. Both studies used a standardized and highly accepted measure of PTSD that elicited Diagnostic and Statistical Manual of Mental Disorders (DSM) criteria.

\section{Assessing trauma}

The responses to trauma were collected in three ways. In all but one study [30], interviewers were used to elicit self-report from youth; unfortunately, there is a reporting bias inherent in self-report, In four studies [24,26-28] measures were delivered by individuals with counseling degrees, who presumably would be more sensitive to assessing traumatic experiences from youth than the officers from the court used to assess trauma exposure in two other studies $[25,29]$. Baglivio and Epps [25] used trauma screening as part of a risk assessment done by an officer of the court to predict the likelihood that the youth would reoffend, and there was no mention of whether the youth were blinded to this, which would further create response bias because youth are less likely to report traumatic history when doing so puts them atrisk to reoffend in the rater's perspective [32]. Lastly, two studies reviewed prior court documentation on the youth (e.g., Child Protective Services reports) to assess past abuse/maltreatment; [28] used this as a supplement to court documentation, and Rawal, et al. [30] used it by itself. King, et al. [28] noted that if there was a discrepancy between the court report and the self-report, they would keep the response that indicated trauma exposure. Allowing the researcher to choose the desired response is also highly problematic.

\section{Type of studies}

I evaluated the studies in this systematic review to determine the strength of the findings. The quality of the study designs was mixed. Of the seven studies, three $[24,29,30]$ stratified their samples to be representative of the state's juvenile justice sample where the research took place in terms of age, sex, race/ethnicity, and legal status. Baglivio and Epps [25] and Lau, et al. [29] used secondary databases containing samples of youth in detention throughout the entire state, allowing for much larger sample sizes. However, these studies are problematic in that Latinx youth were consistently underrepresented in the study samples. In the same two studies, the authors also reported attrition; 22\% in Baglivio and Epps and 5\% in Lau, et al. King, et al. [28] reported that after reviewing the characteristics of the participants they lost, they determined that these participants were more likely to be male or Latinx than the participants who were sampled.

Most of the studies used count data to summarize cumulative traumatic responses. The number of possible count outcomes ranged from 0 to 19; only Abram, et al. [24] used a count regression model to analyze count data. Ford, et al. [27] used an exploratory latent class analysis to determine the number of latent classes of polyvictimization and subsequent PTSD, while two studies used logistic regression to compare rates of abuse by demographic characteristics $[26,28]$. Rawal, et al. [30] used analysis of variance to detect and report differences chi-square tests to report differences, and Baglivio and Epps used prevalence rates.

\section{Trauma rates}

I examined seven studies that assessed either the number of traumatic experiences or PTSD severity by ethnicity/race or Latinx gender. I also investigated traumatic loss by ethnicity/race and sex abuse by Latinx gender. The studies varied in that not all allowed comparisons by trauma experience and PTSD severity. Table 2 shows the effect sizes of traumatic exposure, PTSD severity, traumatic loss, and sex abuse with odds ratios and 95\% confidence intervals.

\section{Trauma exposure by race/ethnicity}

There were mixed findings in terms of rates of trauma exposure by race/ethnicity. In Abram, et al. [24], Latinx youth reported a mean number of traumatic experiences that was higher than that of White youth and comparable to that of Black youth. In Ford, et al. [27], poly victimization and subsequent PTSD were combined using exploratory latent class analysis, which created a high polyvictimization class $(M=11.37, S D=1.11$; trauma types), relatively moderate adversity $(M=8.9, S D$ $=0.34$; trauma types), and low $(M=7.39, S D=0.42$; trauma types). Latinx youth ( $n=18,3 \%$ ) were the lowest proportion of youth in the highest polyvictimization class (White $=5.9 \%$, Black $=5.4 \%$ ); however, in the same study, the proportion of Latinx youth that made up the other two subcategories was similar to White and Black youth at the moderate and low categories of polyvictimization. Baglivio and Epps [25] and Lau, et al. [29] found that Latinx youth reported the lowest number of cumulative trauma exposures. King, et al. [28] report- 


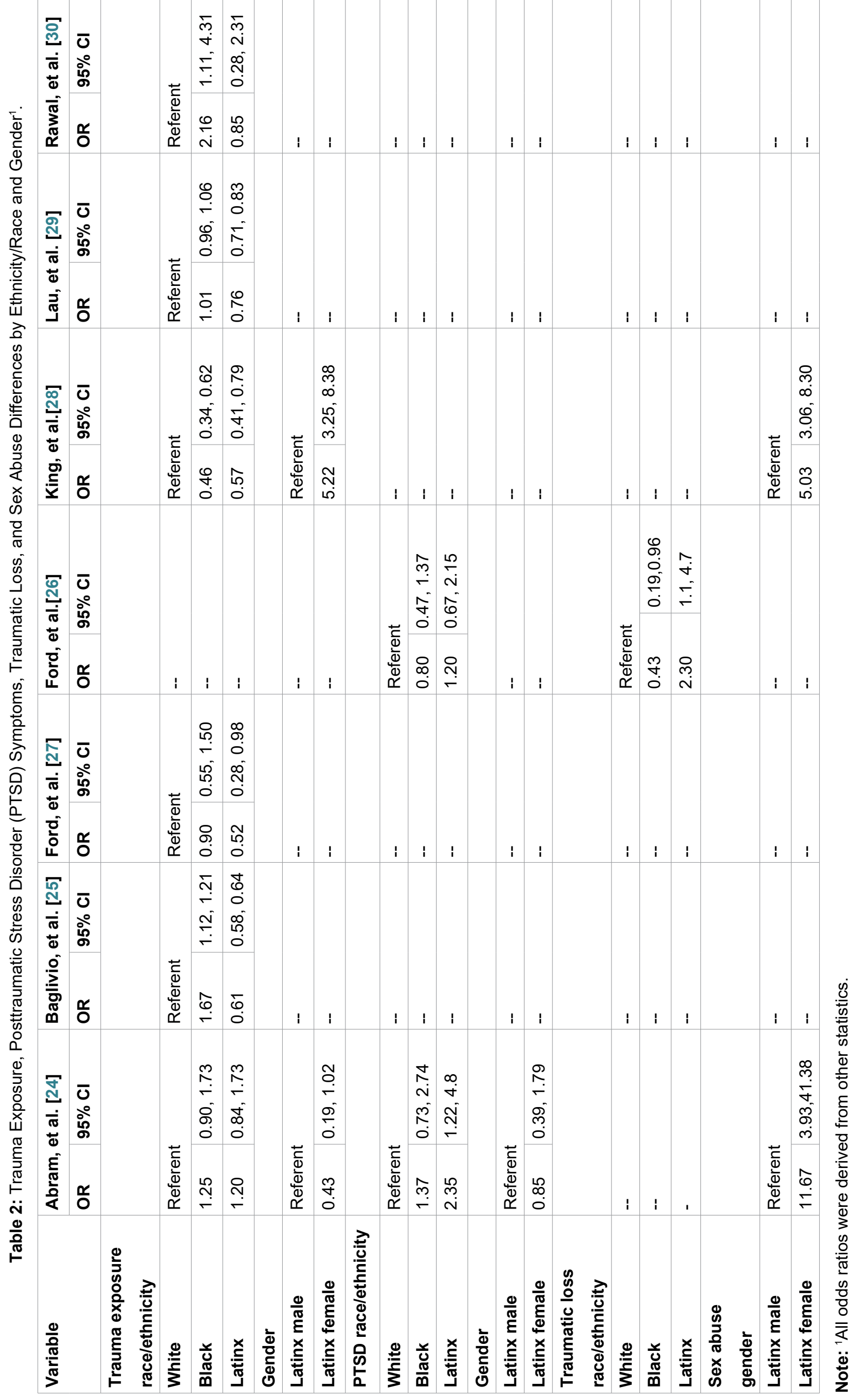


ed that Latinx youth had trauma rates similar to those of Black youth but less than those of White youth. One study with a low $N$ was in conclusive [30]. Lastly, Ford, et al. [26] indicated that Latinx youth, when compared to Black and White youth, were more likely to report both traumatic loss and community violence.

\section{Trauma exposure by latinx gender}

Trauma exposure by Latinx gender was also mixed. Abram, et al. [24] found that a higher proportion of Latinx males than females reported experiencing trauma. King, et al. [28] found that, on every construct of types of abuse (i.e., physical and sexual abuse), a higher proportion of Latinx females disclosed every type compared to Latinx males. Abram, et al. allowed analysis by trauma type and reported that a higher proportion of Latinx males than Latinx females disclosed two items: "witnessing or experiencing someone get hurt badly or killed" and "being threatened by a weapon."Abram, et al. also found that a higher proportion of Latinx females than males disclosed "forced to do something sexual."

\section{PTSD by race/ethnicity}

Abram, et al. [24] and Ford, et al. [26] reported PTSD symptoms by ethnicity/race. Abram, et al. found that Latinx youth disclosed clinically significant PTSD symptoms at a statistically significantly higher rate than did White and Black youth. Ford, et al. reported a similar trend, while not statistically significant, with Latinx youth reporting more severe PTSD symptoms.

\section{PTSD by latinx gender}

Abram, et al. [24] were the only researchers to compare PTSD symptoms by Latinx gender. They found that a higher proportion of Latinx males were diagnosed with clinically significant PTSD than were Latinx females.

\section{Discussion}

My study aim was to assess trauma exposure and severity of PTSD symptoms by race/ethnicity and Latinx gender. I had mixed findings. Latinx youth did report high rates of cumulative trauma, particularly when assessed for traumatic loss and community violence. Only two studies [26] fit the criteria to analyze PTSD symptoms by race/ethnicity; both reported Latinx youth having greater severity of PTSD symptoms than youth of other backgrounds. Latinx females reported higher rates of sexual abuse whereas males reported more witnessing or experiencing violence. Treatment of juvenile justice-involved youth should take these differences into account.

\section{Possible distortions for latinx youth's lower trau- ma rates}

That Latinx youth, in comparison to White and Black detained youth, showed lower rates of trauma experiences but more PTSD severity warrants further explo- ration. A primary reason for this seeming discrepancyis the narrow focus of the elicited trauma exposure of the justice-involved youth. King, et al. [28], Rawal, et al. [30], and Baglivio and Epps [25] solely focused on traumatic experiences from physical or sexual abuse, with Baglivio and Epps also focusing on traumatic experiences endured in the family system. Lau, et al. [29] elicited a limited number of trauma types, and Ford, et al. [27] combined trauma symptoms and PTSD severity, there by not allowing for a detailed examination of trauma exposure. Lau, et al. is also problematic in that the researchers categorized individuals of Caribbean descent as Black. Many Latinx youth hail from Caribbean countries.

\section{Community violence}

Researchers have documented that Latinx youth endure unique and distinct types of trauma compared to White and Black youth. For example, Finkelhor, et al. [33] noted that Latinx youth are more likely to live in disadvantaged neighborhoods and experience community violence compared with youth of other racial/ ethnic groups in the United States. Of the six studies that provided comparisons by trauma experience, only Abram, et al. [24], Ford, et al. [26], and Ford, et al. [27] elicited traumatic experience related to community violence. In these three studies, Latinx youth showed a trend toward a higher mean number of traumatic experiences than White youth, with Abram, et al. also showing a comparable number to Black youth. Latinx youth in Abram, et al. and Ford, et al. [26] also showed more severe PTSD symptoms than White and Black youth.

\section{Trauma in home country}

Phipps and Degges-White [34] identified another distinct type of trauma related to Latinx youth, showing that they are also at risk for poor health outcomes due to traumatic events in their country of origin stemming from political warfare, gang violence, and drug cartels with minimal protection by police and during the migration process. In Ford, et al. [26] study that elicited traumatic separation/loss, Latinx youth were substantially higher compared to both Black and White youth. In addition, in a community sample of 1,004 school-aged youth, Jaycox, et al. [35] noted that $76 \%$ reported exposure to violence in their home countries. However, none of the studies reviewed here elicited trauma experience related to traumatic events in the home country or during migration. In addition, a serious methodological complaint is that every study analyzed Latinx youth as a monolithic ethnic group rather than considering them by country of origin. Trauma measures should be relevant to the lived experiences of Latinx youth.

\section{Traumatic separation and loss}

One last methodological complaint specific to Latinx individuals is the measurement of traumatic separation 
and loss. Traumatic separation and loss was not a construct consistently assessed in studies on traumatic exposure reviewed for this study. Given the propensity to immigration in comparison to White and Black youth, this is a construct that should be routinely assessed.

\section{Gender differences}

Gender differences of justice-involved youth have been a research focus [18]. Latinx girls involved in the justice system are on the rise as onefourth of all Latinx arrests are female [36]. However, there has been limited research on justice-involved Latinx females [18]. Many justice-involved girls have trauma histories with resulting unmet behavioral health needs [37]. My findings are consistent with the literature in that there was limited attention given to gender differences in the studies reviewed, particularly related to Latinx youth and gender. However, when sexual abuse was taken into account and assessed by both gender and race/ethnicity, Latinx girls consistently demonstrated higher rates. Further research is needed to appropriately assess and intervene for justice-involved Latinx girls.

\section{Limitations and Future Research}

This systematic review should be considered with some limitations. First, I found only a small number of studies that fit the criteria for this review. Second, given the lack of construct equivalence in measuring traumatic experiences, the generalizability of this study related to traumatic experiences and potential race/ethnic differences and gender differences is limited. Lastly, only two studies compared traumatic experiences by Latinx gender [28]. Although the lack of construct equivalence and Latinx gender comparisons are problematic, public health officials and policy makers can use these findings to further inform proper assessment of Latinx youth, which will provide proper referral strategies for early prevention and intervention efforts.

Future research would benefit from the aforementioned study limitations. Specifically, comparing traumatic experiences of justice-involved youth by race/ ethnicity and gender, taking into account Latinx youth's specific traumas endured in the home country and during migration, traumatic separation and loss due to migration, and community violence. Furthermore, comparing traumatic experiences by Latinx gender would further assist practitioners seeking to properly refer justice-involved youth to the appropriate services. Lastly, assessing whether proper intake of traumatic experiences benefits treatment outcomes is recommended.

\section{Conclusion}

Latinx youth involved in the justice system have been shown to endorse high rates of trauma exposure [20]. Trauma exposure has been linked to unmet behavioral health needs (i.e., psychiatric symptoms, substance use, and sexual risk behaviors), and unmet behavioral health needs have been linked to recidivism. I set out to compare traumatic experiences of justice-involved youth by race/ethnicity and gender. I found that the lack of construct equivalence when eliciting traumatic experiences led to variability in trauma rates and ultimately resulted in misclassification of trauma rates. In order to provide proper assessment and intervention to offset further justice involvement and to treat the unmet behavioral health needs of justice-involved Latinx youth, clinicians are urged to use measures of trauma experience and to take these findings from these measures into consideration.

\section{Disclosure of Interest}

This author has no disclosures of interest.

\section{Funding}

This study was funded by National Institute of Drug Abuse under grant number RO1DA034538-05S1 and the National Institute of Health Loan Repayment Program, NIMHD (Hoskins, L60 MD009257).

\section{Acknowledgements}

The author extends his gratitude to Peter Bacchetti, $\mathrm{PhD}$ and Megan Morris, PhD, (UCSF Department of Epidemiology and Biostatistics), and Marina Tolou-Shams, PhD (UCSF, Department of Psychiatry and Weill Institute for Neurosciences) for their assistance with their feedback on the manuscript and data analysis.

\section{Authors Declarations}

\section{Ethics approval and consent to participate}

Not applicable.

\section{Consent for publication}

Not applicable.

\section{Availability of data and materials}

Names of articles for this review are referenced in this article and can be available upon request.

\section{Competing interest}

The author certifies that he has NO affiliations with or involvement in any organization or entity with any financial interest (such as honoraria; educational grants; participation in speakers' bureaus; membership, employment, consultancies, stock ownership, or other equity interest; and expert testimony or patent-licensing arrangements), or non-financial interest (such as personal or professional relationships, affiliations, knowledge or beliefs) in the subject matter or materials discussed in this manuscript.

\section{Authors' contributions}

This author completed all aspects of the article.

\section{References}

1. McClelland GM, Teplin LA, Abram KM (2004) Detection 
and prevalence of substance use among juvenile detainees (NCJ 203934). Office of Juvenile Justice and Delinquency Prevention, U.S. Department of Justice, Office of Justice Programs.

2. Winkelman TN, Frank JW, Binswanger IA, Pinals DA (2017) Health conditions and racial differences among justice-involved adolescents, 2009 to 2014. Academic Pediatrics 17: 723-731.

3. Coalition for Juvenile Justice (2001) ¡Esperanza! Awakening to the strength of Latino youth. IssueLab.

4. Human Rights Watch (2002) Race and incarceration in the United States [Press backgrounder].

5. Children's Bureau (2015) Child maltreatment 2015. U.S. Department of Health and Human Services, Administration for Children and Families, Administration on Children, Youth and Families.

6. Kemp K, Thamortharan S, Poindexter B, Barker D, Tolou-Shams M, et al. (2017) Emotion regulation as a predictor of juvenile arrest. Criminal Justice and Behavior 44: 912-926.

7. Schubert CA, Mulvey EP, Glasheen C (2011) Influence of mental health and substance use problems and criminogenic risk on outcomes in serious juvenile offenders. Journal of the American Academy of Child \& Adolescent Psychiatry 50: 925-937.

8. Abram KM, Teplin LA, King DC, Longworth SL, Emanuel $\mathrm{KM}$, et al. (2013) PTSD, trauma, and comorbid psychiatric disorders in detained youth.Office of Juvenile Justice and Delinquency Prevention, U. S. Department of Justice, Office of Justice Programs.

9. Yonek JC, Dauria EF, Kemp K, Koinis-Mitchell D, Marshall BDL, et al. (2019) Factors associated with use of mental health and substance use treatment services by justice-involved adolescents. Psychiatric Services 70: 586-595.

10. Substance Abuse and Mental Health Services Administration (2014) TIP 57: Trauma-informed care in behavioral health services (SMA14-4816).

11. Briere J, Scott C (2015) Principles of trauma therapy: A guide to symptoms, evaluation, and treatment ( $2^{\text {nd }}$ edn), Sage Publications.

12. van der Kolk BA (2014) The body keeps the score: Brain, mind, and body in the healing of trauma. Viking.

13. Husarewycz MN, El-Gabalawy R, Logsetty S, Sareen J (2014) The association between number and type of traumatic life experiences and physical conditions in a nationally representative sample. General Hospital Psychiatry 36: 26-32.

14. Crenshaw $K$ (1995) The intersection of race and gender. In: Crenshaw K, Gotanda N, Peller G, Thomas K, Critical race theory: The key writings that formed the movement. The New Press, 357-383.

15. Hooks B (1990) Yearning: Race, gender, and cultural politics. South End Press.

16. Karnik NS, Soller MV, Redlich A, Silverman MA, Kraemer $\mathrm{HC}$, et al. (2010) Prevalence differences of psychiatric disorders among youth after nine months or more of incarceration by race/ethnicity and age. Journal of Health Care for the Poor and Underserved 21: 237-250.

17. Racz SJ, Saha S, Trent M, Adger H, Bradshaw CP, et al. (2015) Polysubstance use among minority adolescent males incarcerated for serious offenses. Child Youth Care Forum 45: 205-222.
18. Lopez V, Nuño L (2016) Latina and African-American females in the juvenile justice system: Needs, problems, and solutions. Sociology Compass 10: 24-37.

19. Abram KM, Zwecker NA, Welty LJ, Hershfield JA, Dulcan MK, et al. (2015) Comorbidity and continuity of psychiatric disorders in youth after detention: A prospective longitudinal study. JAMA Psychiatry 72: 84-93.

20. Hoskins D, Tolou-Shams M, Marshall B, Koinis-Mitchell D, Galbriath K (2019) Latinx youth in first contact with the justice system: Trauma and associated behavioral health needs. Child Psychiatry and Human Development 50: 459472.

21. Griner D, Smith T (2006) Culturally adapted mental health intervention: A meta-analytic review. Psychotherapy: Theory, Research, Practice, Training 43: 531-548.

22. White C (2016) Incarcerating youth with mental health problems: A focus on the intersection of race, ethnicity, and mental illness. Youth Violence and Juvenile Justice 14: 426-447.

23. Hinton DE, Pich V, Hofmann SG, Otto MW (2013) Acceptance and mindfulness techniques as applied to refugee and ethnic minority populations with PTSD: Examples from "culturally adapted CBT." Cognitive and Behavioral Practice 20: 33-46.

24. Abram KM, Teplin LA, Charles DR, Longworth SL, McClelland GM, et al. (2004) Post-traumatic stress disorder and trauma in youth in juvenile detention. Archives of General Psychiatry 61: 403-410.

25. Baglivio MT, Epps N (2016) The interrelatedness of adverse childhood experiences among high-risk juvenile offenders. Youth Violence and Juvenile Justice 14: 179-198.

26. Ford J, Hartman K, Hawke J, Chapman J (2008) Traumatic victimization, posttraumatic stress disorder, suicidal ideation, and substance abuse risk among juvenile justice-involved youth. Journal of Child \& Adolescent Trauma 1: 7592.

27. Ford J, Grasso D, Hawke J, Chapman J (2013) Poly-victimization among juvenile justice-involved youths. Child Abuse \& Neglect 37: 788-800.

28. King D, Abram K, Romer E, Washburn J, Welty L, et al. (2011) Childhood maltreatment and psychiatric disorders among detained youths. Psychiatric Services 62: 14301438.

29. Lau K, Aalsma M, Holloway E, Wiehe S, Vachon D (2015) The effects of racial heterogeneity on mental health: $A$ study of detained youth across multiple counties. American Journal of Orthopsychiatry 85: 421-430.

30. Rawal P, Romansky J, Jenuwine M, Lyons J (2004) Racial differences in the mental health needs and service utilization of youth in the juvenile justice system. The Journal of Behavioral Health Services \& Research 31: 242-254.

31. Wilson DB (n.d.) Practical meta-analysis effect size calculator [Online calculator].

32. van Batenburg-Eddes $T$, Butte $D$, van de Looij-Jansen $P$, Schiethart W, Raat H, et al. (2012) Measuring juvenile delinquency: How do self-reports compare with official police statistics? European Journal of Criminology 9: 23-37.

33. Finkelhor D, Shattuck A, Turner HA, Hamby SL (2014) The lifetime prevalence of child sexual abuse and sexual assault assessed in late adolescence. The Journal of Adolescent Health 55: 329-333.

34. Phipps RM, Degges-White S (2014) A new look at trans- 
generational and trauma transmission: Second-generation Latino immigrant youth. Journal of Multicultural Counseling and Development 42: 174-187.

35. Jaycox LH, Stein BD, Kataoka SH, Wong W, Fink A, et al. (2002) Violence exposure, posttraumatic stress disorder, and depressive symptoms among recent immigrant schoolchildren. Journal of the American Academy of Adolescent Psychiatry 41: 1104-1110.
36. Hockenberry S, Puzzanchera C (2016) Delinquency cases involving Hispanic youth, 2013 (NCJ 249915). Office of Juvenile Justice and Delinquency Prevention, U.S. Department of Justice, Office of Justice Programs.

37. Belenko S, Langley S, Crimmins S, Chaple M (2004) HIV risk behaviors, knowledge, and prevention education among offenders under community supervision: A hidden risk group. AIDS Education and Prevention 16: 367-385. 